\title{
Factors Associated with Inadequate Intravenous Colistin Dosages: Post Hoc Analysis of a Multicenter, Cross-Sectional Study
}

\author{
Daniele Roberto Giacobbe ${ }^{1,2, *}$, Michele Mirabella ${ }^{2}$, Matteo Rinaldi ${ }^{3}$, Angela Raffaella Losito ${ }^{4}$, \\ Francesca Raffaelli ${ }^{4}$, Filippo Del Puente ${ }^{1,5}$, Carolina Saffioti ${ }^{6}\left(\mathbb{D}\right.$, Malgorzata Mikulska ${ }^{1,2}(\mathbb{D}$, \\ Maddalena Giannella ${ }^{3}$, Pierluigi Viale ${ }^{3}$, Mario Tumbarello ${ }^{7}$, Matteo Bassetti ${ }^{1,2}$ and on behalf of SITA GIOVANI \\ (Young Investigators Group of the Società Italiana Terapia Antinfettiva) and the COLI-CROSS Study Group ${ }^{\dagger}$
}

check for

updates

Citation: Giacobbe, D.R.; Mirabella, M.; Rinaldi, M.; Losito, A.R.; Raffaelli, F.; Del Puente, F.; Saffioti, C.;

Mikulska, M.; Giannella, M.; Viale, P.; et al. Factors Associated with Inadequate Intravenous Colistin Dosages: Post Hoc Analysis of a Multicenter, Cross-Sectional Study. Antibiotics 2021, 10, 1554. https:// doi.org/10.3390/antibiotics10121554

Academic Editor:

Ágnes Pál-Sonnevend

Received: 30 November 2021 Accepted: 17 December 2021 Published: 19 December 2021

Publisher's Note: MDPI stays neutral with regard to jurisdictional claims in published maps and institutional affiliations.

Copyright: (C) 2021 by the authors Licensee MDPI, Basel, Switzerland. This article is an open access article distributed under the terms and conditions of the Creative Commons Attribution (CC BY) license (https:/ / creativecommons.org/licenses/by/ $4.0 /)$.
1 Department of Health Sciences (DISSAL), University of Genoa, 16132 Genoa, Italy; filippo.del.puente@galliera.it (F.D.P.); m.mikulska@unige.it (M.M.); matteo.bassetti@unige.it (M.B.)

2 Clinica Malattie Infettive, IRCCS Ospedale Policlinico San Martino, 16132 Genoa, Italy; michelemirabella@hsanmartino.it

3 Operative Unit of Infectious Diseases, IRCCS Azienda Ospedaliero-Universitaria di Bologna, 40138 Bologna, Italy; mat.rinaldi1989@gmail.com (M.R.); maddalena.giannella@unibo.it (M.G.); pierluigi.viale@unibo.it (P.V.)

4 Dipartimento di Scienze di Laboratorio e Infettivologiche, Fondazione Policlinico Universitario A. Gemelli IRCCS, 00168 Roma, Italy; lositoraffaella@yahoo.it (A.R.L.); franraffaelli@gmail.com (F.R.)

5 Department of Infectious Diseases, Galliera Hospital, 16128 Genoa, Italy

6 Infectious Diseases Unit, IRCCS Istituto Giannina Gaslini, 16147 Genoa, Italy; carolinasaffioti@gaslini.org

7 Department of Medical Biotechnologies, University of Siena, 53100 Siena, Italy; mario.tumbarello@unisi.it

* Correspondence: danieleroberto.giacobbe@unige.it; Tel.: +39-010-555-4654; Fax: +39-010-555-6712

+ Membership of the COLI-CROSS Study Group is provided in the Acknowledgments.

Abstract: Colistin is a last-resort agent for the treatment of infections due to Gram-negative bacteria with difficult-to-treat resistance. The primary objective of this post hoc analysis of a cross-sectional study conducted in 22 Italian hospitals was to assess factors associated with inadequate intravenous colistin dosage. Overall, 187 patients receiving intravenous colistin were included in the analyses. Inadequate colistin dosages were administered in $27 \%$ of cases (50/187). In multivariable analysis, AKI (dummy variable with KDIGO stage 0 as a reference, odds ratio (OR) 3.98 with $95 \%$ confidence interval (CI) 1.48-10.74 for stage 1, OR 4.44 with 95\% CI 1.17-16.93 for stage 2, OR 9.41 with $95 \%$ CI 1.59-55.70 for stage 3; overall $p=0.001$ ) retained an independent association with inadequate colistin dosage, whereas the presence of a central venous catheter was associated with adequate colistin dosage (OR: 0.34 for inadequate dosage, 95\% CI: $0.16-0.72, p=0.004$ ). These results were confirmed in an additional multivariable model with the center as a random effect. The association between AKI and inadequate dosage may reflect the perception of an increased risk of nephrotoxicity in patients with impaired renal function, which nonetheless should not be accompanied by dosage reductions beyond those recommended and could represent the target of dedicated antimicrobial stewardship efforts.

Keywords: colistin; dosage; polymyxin; antimicrobial stewardship; acute kidney injury

\section{Introduction}

Colistin is a last-resort agent for the treatment of infections due to Gram-negative bacteria with difficult-to-treat resistance, especially carbapenem-resistant Acinetobacter baumannii, carbapenem-resistant Enterobacterales, and carbapenem-resistant Pseudomonas aeruginosa [1-4].

Although an important reduction in colistin use has been registered in the past two years, owing to the availability of novel, less nephrotoxic, and possibly more efficacious 
beta-lactams and beta-lactam/beta-lactamase inhibitor combinations, in selected cases (e.g., allergy or resistance to novel agents), colistin may still represent a precious lastresort treatment option for infections due to carbapenem-resistant Gram-negative bacteria; thus, our efforts to optimize its use and efficacy in real-world practice should still not be abandoned [4-10].

In the present post hoc analysis of a previous large multicenter study describing the use of colistin in Italian hospitals [11], we aimed to identify modifiable predictors of inadequate intravenous colistin dosage that could represent the selected target of future dedicated antimicrobial stewardship interventions.

\section{Methods}

This is a post hoc analysis of a multicenter, cross-sectional study describing colistin use in 22 Italian hospitals (COLI-CROSS study) [11]. The primary objective of this post hoc analysis was to assess factors associated with inadequate intravenous colistin dosage. All patients included in the main study who received intravenous colistin were included. In line with the lack of detailed dosage information in patients receiving hemodialysis in the COLI-CROSS study, patients on hemodialysis were excluded from this post hoc analysis. Inadequate colistin dosage was defined as the absence of an adequate loading dose (i.e., 9 million units of colistimethate) and/or the absence of adequate maintenance dosages according to the Committee for Medicinal Products for Human Use of the European Medicines Agency, adjusted to renal function (see supplementary material) [12]. The COLI-CROSS study was approved by the Ethics Committee of the coordinating center (Liguria Region Ethics Committee, registry number 321/2017). The other participating centers followed the local ethical requirements. The study was conducted according to the guidelines of the Declaration of Helsinki.

\section{Statistical Analysis}

The primary study analysis was the identification of factors associated with inadequate colistin dosage. To this aim, demographic and clinical variables were tested for their association with inadequate colistin dosage in univariable logistic regression models. Then, variables potentially associated with inadequate dosage in univariable models $(p<0.10)$ were included in an initial multivariable logistic regression model, and further selected for the final model (model A) using a stepwise backward procedure. The variables included in model A were additionally tested for their association with inadequate colistin dosage in a generalized, linear mixed model (model B, with logit as the link function and center as a random effect). The analyses were performed using $R$ Statistical Software version 3.5.2 ( $R$ Foundation for Statistical Computing, Vienna, Austria). The mixed model was built with the glmer function in the lme4 package for R Statistical Software.

\section{Results}

Overall, 187 patients were included in the analysis (Figure 1). The first two columns of Table 1 show the demographic and clinical characteristics of patients who received adequate colistin dosages and those who receive inadequate colistin dosages. Overall, 50/187 patients $(27 \%)$ received inadequate colistin dosages (lower than those recommended). Table 1 also shows the results of the univariable analysis of factors potentially associated with the administration of an inadequate colistin dosage. Increasing age, chronic renal failure, and acute kidney injury (AKI) were associated with inadequate colistin dosage in univariable comparisons, whereas presence of a central venous catheter was associated with adequate colistin dosage. 


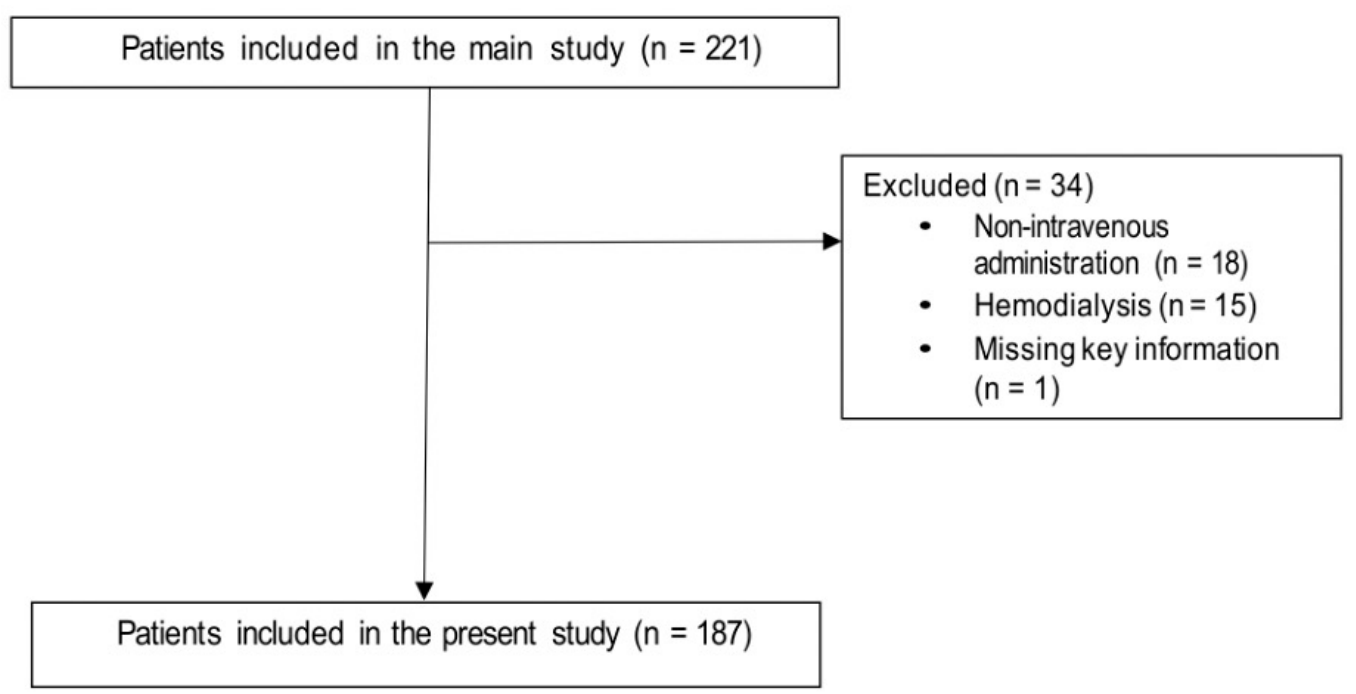

Figure 1. Flow-chart of the patient selection process.

Table 1. Characteristics of the study population and univariable analysis of factors associated with inadequate intravenous colistin dosage.

\begin{tabular}{|c|c|c|c|c|}
\hline Variable & $\begin{array}{c}\text { Patients Receiving Inadequate } \\
\text { Dosage }(\%) \\
(n=50)\end{array}$ & $\begin{array}{c}\text { Patients Receiving } \\
\text { Adequate Dosage (\%) } \\
\qquad(n=137)\end{array}$ & OR $(95 \%$ CI $)$ & $p$ \\
\hline Age in years, median IQR & $69(53-80)$ & $61(48-71)$ & $1.03(1.00-1.05)$ & 0.021 \\
\hline Male gender & $32(64)$ & $81(59)$ & $1.23(0.63-2.40)$ & 0.547 \\
\hline Previous treatment with colistin & $8(16)$ & $20(15)$ & $1.11(0.46-2.72)$ & 0.812 \\
\hline $\begin{array}{l}\text { Hospital stay before colistin } \\
\text { initiation in days, median (IQR) }\end{array}$ & $17(4-40)$ & $23(13-50)$ & $1.00(0.99-1.01)$ & 0.960 \\
\hline ICU stay & $22(44)$ & $56(41)$ & $1.13(0.59-2.19)$ & 0.701 \\
\hline Diabetes mellitus & $15(30)$ & $27(20)$ & $1.75(0.84-3.65)$ & 0.138 \\
\hline Chronic renal failure & $5(10)$ & $3(2)$ & $4.96(1.14-21.60)$ & 0.033 \\
\hline Solid neoplasm & $10(20)$ & $23(17)$ & $1.24(0.54-2.83)$ & 0.611 \\
\hline Hematological malignancy & $2(4)$ & $13(9)$ & $0.40(0.09-1.83)$ & 0.236 \\
\hline Charlson score, median (IQR) & $2(1-3)$ & $2(1-3)$ & $1.12(0.97-1.29)$ & 0.136 \\
\hline Presence of CVC & $30(60)$ & $109(80)$ & $0.39(0.19-0.78)$ & 0.008 \\
\hline Presence of urinary catheter & $39(78)$ & $113(82)$ & $0.75(0.34-1.68)$ & 0.488 \\
\hline Mechanical ventilation & $18(36)$ & $37(27)$ & $1.52(0.76-3.03)$ & 0.234 \\
\hline Neutropenia & $2(4)$ & $11(8)$ & $0.48(0.10-2.23)$ & 0.347 \\
\hline Septic shock & $12(24)$ & $27(20)$ & $1.29(0.59-2.79)$ & 0.523 \\
\hline Pulmonary infection & $15(30)$ & $44(32)$ & $0.91(0.45-1.83)$ & 0.783 \\
\hline KDIGO stage of AKI & & & & 0.002 \\
\hline No AKI & $31(62)$ & $120(88)$ & (ref) & \\
\hline Stage 1 & $10(20)$ & $10(7)$ & $3.87(1.48-10.12)$ & \\
\hline Stage 2 & $5(10)$ & $5(4)$ & $3.87(1.05-14.22)$ & \\
\hline Stage 3 & $4(8)$ & $2(1)$ & $7.74(1.36-44.23)$ & \\
\hline Type of prescriber $\S$ & & & & 0.346 \\
\hline Infectious diseases specialist & $36(72)$ & $109(81)$ & (ref) & \\
\hline Intensive care specialist & $10(20)$ & $16(12)$ & $1.89(0.79-4.54)$ & \\
\hline Others $\$ \S$ & $4(8)$ & $9(7)$ & $1.35(0.39-4.63)$ & \\
\hline Targeted therapy $\S \S \S$ & $40(80)$ & $101(74)$ & $1.43(0.65-3.14)$ & 0.379 \\
\hline Combination therapy & $42(84)$ & $108(79)$ & $1.41(0.60-3.33)$ & 0.434 \\
\hline
\end{tabular}

Results are presented as $\mathrm{n}(\%)$ unless otherwise indicated. AKI, acute kidney injury; CI, confidence intervals; CVC, central venous catheter; ICU, intensive care unit; IQR, interquartile range; KDIGO, Kidney Disease: Improving Global Outcomes; OR, odds ratio. ${ }^{\S}$ Information missing for $3 / 187$ patients $(2 \%)$. $\$ \S$ Hematologist $(n=7)$, pneumologist $(n=3)$, internist $(n=1)$, neurologist $(n=1)$, surgeon $(n=1)$. $\$ \$ \$$ With regard to carbapenem-resistant Acinetobacter baumannii (CRAB), carbapenem-resistant Enterobacterales (CRE), and carbapenem-resistant Pseudomonas aeruginosa (CRPA): CRAB $(\mathrm{n}=60), \mathrm{CRE}(\mathrm{n}=38), \mathrm{CRPA}(\mathrm{n}=24), \mathrm{CRAB}$ plus $\mathrm{CRE}(\mathrm{n}=11), \mathrm{CRE}$ plus $\mathrm{CRPA}(\mathrm{n}=5), \mathrm{CRAB}$ plus CRPA $(n=2)$, CRAB plus CRE plus CRPA $(n=1)$.

The results of the multivariable analysis of factors associated with inadequate colistin dosages are shown in Table 2. AKI (dummy variable with KDIGO stage 0 as reference, odds ratio [OR] 3.98 with 95\% confidence intervals [CI] 1.48-10.74 for stage 1, OR 4.44 with 
95\% CI 1.17-16.93 for stage 2, OR 9.41 with 95\% CI 1.59-55.70 for stage 3; overall $p$ for the dummy variable 0.001) retained an independent association with inadequate colistin dosages, whereas presence of a central venous catheter was independently associated with adequate colistin dosages (OR 0.34 for inadequate dosages, 95\% CI 0.16-0.72, $p=0.004$ ). These results were confirmed in the additional multivariable model with the center as a random effect (model B, also shown in Table 2).

Table 2. Multivariable analysis of factors associated with inadequate intravenous colistin dosage *.

\begin{tabular}{ccc}
\hline Model A (AIC 204.5) & OR $(\mathbf{9 5} \% \mathbf{C I})$ & $p$ \\
\hline Presence of CVC & $0.34(0.16-0.72)$ & $0.004 \S$ \\
KDIGO stage of AKI & $($ ref $)$ & $0.001 \S$ \\
No AKI & $3.98(1.48-10.74)$ & \\
Stage 1 & $4.44(1.17-16.93)$ & \\
Stage 2 & $9.41(1.59-55.70)$ & $p$ \\
Stage 3 & OR (95\% CI) & $0.008 \S$ \\
\hline Model B ** (AIC 199.4) & $0.33(0.15-0.75)$ & $0.002 \S$ \\
\hline Presence of CVC & $($ ref) & \\
KDIGO stage of AKI & $4.73(1.56-14.37)$ & \\
No AKI & $4.83(105-22.22)$ & \\
Stage 1 & $8.23(1.20-56.64)$ & \\
Stage 2 &
\end{tabular}

AIC, Akaike information criterion; AKI, acute kidney injury; CI, confidence intervals; CVC, central venous catheter; OR, odds ratio. * Only variables retained in the final multivariable model after stepwise backward selection are shown in the table. ${ }^{* *}$ Model B included center as a random intercept (variance 0.696, standard deviation 0.834). $\S_{p}<0.05$.

\section{Discussion}

The COLI-CROSS study overall registered high rates of adequate colistin dosages (79\% for loading dosages and $85 \%$ for maintenance dosages, respectively) [11]. Nonetheless, a nonnegligible proportion of patients (21\%) still did not receive a proper loading dose, a fact that prompted the conduction of the present post hoc analysis. Indeed, the identification of possible modifiable independent predictors of inadequate dosages could prompt dedicated antimicrobial stewardship interventions.

In our analysis, two factors showed an independent association with either inadequateness or adequateness of intravenous colistin dosages. On the hand, AKI was associated with an increased risk of receiving inadequate colistin dosages. In this regard, while it is well known that colistin is nephrotoxic, the perceived risk of nephrotoxicity should not lead clinicians to further reduce colistin dosages beyond the standard adjustments recommended by guidelines since this could unacceptably increase the risk of suboptimal colistin concentrations at the site of infection, especially for pneumonia $[4,8,13]$. On the other hand, the presence of a central venous catheter was independently associated with adequate colistin dosage in our analysis. The exact reason for this potential protective effect remains somewhat elusive, although some possible non-mutually exclusive explanations are the following: (i) difficulty to administer all required doses in some patients without a central catheter and concomitant difficult peripheral venous access; (ii) the presence of a higher proportion of patients with a central venous catheter in intensive care units, where infections by carbapenem-resistant Gram-negative bacteria may be more frequent, and consequently, there could more frequent and confident administration of adequate colistin dosages. In this regard, it is of note that, although not statistically significant, the direction of the association between prescription of by infectious diseases specialists and adequateness of colistin dosages was towards improved adequateness, that may support the need of expertise in prescribing this last resort agent. 
The present study has some important limitations. Besides the inability to assess the adequateness of dosages in peculiar populations such as in patients on hemodialysis owing to the lack of detailed information (see study methods), it is of note that we assessed the adequateness of dosages based on the standards at the time of the study, whereas the international consensus guidelines on the optimal use of the polymyxins released subsequently suggest the possible need of further increasing manteinance dosages in patients with augmented renal clearance [4]. Finally, the lack of therapeutic drug monitoring is an inherent limitation of most large real-life studies on colistin use since it is usually limited to few hospitals and laboratories [13].

In conclusion, we found an association between AKI and inadequate dosage that may reflect the perception of an increased risk of nephrotoxicity in patients with impaired renal function, which nonetheless should not be accompanied by dosage reductions beyond those recommended and could represent the target of dedicated antimicrobial stewardship efforts, together with the proper administration of all required colistin doses.

Supplementary Materials: The following are available online at https:/ /www.mdpi.com/article/10 .3390/antibiotics10121554/s1, File S1: adequate colistin dosage in patients not undergoing hemodialysis according to recommendations at the time of the study.

Author Contributions: Conceptualization, D.R.G., and M.B.; methodology, D.R.G.; formal analysis, D.R.G. and M.M. (Michele Mirabella); data curation, D.R.G., Michele Mirabella, M.R., A.R.L., F.R., F.D.P., C.S.; writing—original draft preparation, D.R.G.; writing—review and editing, D.R.G., Michele Mirabella, M.R., A.R.L., F.R., F.D.P., C.S., M.G., M.M. (Malgorzata Mikulska), P.V., M.T., and M.B.; supervision, D.R.G., and M.B. All authors have read and agreed to the published version of the manuscript.

Funding: The original COLI-CROSS study was partially supported by a grant from the Società Italiana di Terapia Antinfettiva ('Borsa di Studio S.I.T.A. per ricerche su infezioni batteriche e fungine'). The present post-hoc analysis was not funded.

Institutional Review Board Statement: The COLI-CROSS study was approved by the Ethics Committee of the coordinating center (Liguria Region Ethics Committee, registry number 321/2017). The other participating centers followed the local ethical requirements. The study was conducted according to the guidelines of the Declaration of Helsinki.

Informed Consent Statement: All conscious patients signed informed consent to participate in the study. A waiver of informed consent for unconscious patients was obtained in most participating centres (only five unconscious patients were not enrolled).

Data Availability Statement: The data presented in this study are available on reasonable request from the corresponding author.

Acknowledgments: We would like to thank the SITA GIOVANI group. COLI-CROSS Study Group Contributors: C. Aurilio, C. Bolla, S. Boni, G. Borgia, N. Carannante, G. Cassola, G. Ceccarelli, S. Corcione, D. Dalla Gasperina, F.G. De Rosa, C. Dentone, S. Di Bella, N. Di Lauria, M. Feasi, M. Fiore, S. Fossati, E. Franceschini, A. Gori, G. Granata, S. Grignolo, P.A. Grossi, G. Guadagnino, F. Lagi, A.E. Maraolo, V. Marinò, M. Mazzitelli, A. Mularoni, A. Oliva, M.C. Pace, A. Parisini, F. Patti, N. Petrosillo, V. Pota, M. Rossi, A. Santoro, C. Tascini, C. Torti, E.M. Trecarichi, M. Venditti, A. Signori, V. Del Bono, F. Alessandri, A. Alfieri, R.M. Antonello, S. Argentero, S. Artioli, M. Bartoletti, A. Bartoloni, S. Beltramini, D. Bianchi, I. Bisso, E. Blasi Vacca, S. Buono, A.R. Buonomo, M. Canepa, A. Capone, G. Chichino, G. De Benedectis, G. Di Caprio, Z. Di Rosa, F. Di Zazzo, I. Gentile, G. Greco, T. Lupia, R. Luzzati, A. Milano, S. Mornese Pinna, C. Mussini, M.B. Passavanti, C. Rogati, A. Russo, P. Sansone, G. Sarteschi, F. Serapide, M. Spaziante, F. Taglietti, S. Tedeschi, R.F. Tobaldi.

Conflicts of Interest: Outside the submitted work, Daniele Roberto Giacobbe reports investigatorinitiated grants by Pfizer Inc. and Gilead Italia. Outside the submitted work, Matteo Bassetti has received funding for scientific advisory boards, travel and speaker honoraria from Angelini, Astellas, Bayer, BioMérieux, Cidara, Cipla, Gilead, Menarini, MSD, Pfizer, Shionogi, Tetraphase, Nabriva. The other authors did not report conflicts of interest relevant to this paper. 


\section{References}

1. Giacobbe, D.R.; Mikulska, M.; Viscoli, C. Recent advances in the pharmacological management of infections due to multidrugresistant Gram-negative bacteria. Expert Rev. Clin. Pharmacol. 2018, 11, 1219-1236. [CrossRef] [PubMed]

2. Karaiskos, I.; Souli, M.; Galani, I.; Giamarellou, H. Colistin: Still a lifesaver for the 21st century? Expert Opin. Drug Metab. Toxicol. 2017, 13, 59-71. [CrossRef] [PubMed]

3. Nang, S.C.; Azad, M.A.K.; Velkov, T.; Zhou, Q.T.; Li, J. Rescuing the Last-Line Polymyxins: Achievements and Challenges. Pharmacol. Rev. 2021, 73, 679-728. [CrossRef] [PubMed]

4. Tsuji, B.T.; Pogue, J.M.; Zavascki, A.P.; Paul, M.; Daikos, G.L.; Forrest, A.; Giacobbe, D.R.; Viscoli, C.; Giamarellou, H.; Karaiskos, I.; et al. International Consensus Guidelines for the Optimal Use of the Polymyxins: Endorsed by the American College of Clinical Pharmacy (ACCP), European Society of Clinical Microbiology and Infectious Diseases (ESCMID), Infectious Diseases Society of America (IDSA), International Society for Anti-infective Pharmacology (ISAP), Society of Critical Care Medicine (SCCM), and Society of Infectious Diseases Pharmacists (SIDP). Pharmacotherapy 2019, 39, 10-39. [CrossRef] [PubMed]

5. Giacobbe, D.R.; di Masi, A.; Leboffe, L.; Del Bono, V.; Rossi, M.; Cappiello, D.; Coppo, E.; Marchese, A.; Casulli, A.; Signori, A.; et al. Hypoalbuminemia as a predictor of acute kidney injury during colistin treatment. Sci. Rep. 2018, 8, 11968. [CrossRef] [PubMed]

6. $\quad$ Bassetti, M.; Labate, L.; Russo, C.; Vena, A.; Giacobbe, D.R. Therapeutic options for difficult-to-treat Acinetobacter baumannii infections: A 2020 perspective. Expert Opin. Pharm. 2021, 22, 167-177. [CrossRef] [PubMed]

7. Karaiskos, I.; Galani, I.; Papoutsaki, V.; Galani, L.; Giamarellou, H. Carbapenemase producing Klebsiella pneumoniae: Implication on future therapeutic strategies. Expert Rev. Anti. Infect. Ther. 2021, 19, 1-17. [CrossRef] [PubMed]

8. Nation, R.L.; Forrest, A. Clinical Pharmacokinetics, Pharmacodynamics and Toxicodynamics of Polymyxins: Implications for Therapeutic Use. Adv. Exp. Med. Biol. 2019, 1145, 219-249. [CrossRef] [PubMed]

9. Nation, R.L.; Garonzik, S.M.; Thamlikitkul, V.; Giamarellos-Bourboulis, E.J.; Forrest, A.; Paterson, D.L.; Li, J.; Silveira, F.P. Dosing guidance for intravenous colistin in critically-ill patients. Clin. Infect. Dis. 2017, 64, 565-571. [CrossRef] [PubMed]

10. Nation, R.L.; Rigatto, M.H.P.; Falci, D.R.; Zavascki, A.P. Polymyxin Acute Kidney Injury: Dosing and Other Strategies to Reduce Toxicity. Antibiotics 2019, 8, 24. [CrossRef] [PubMed]

11. Giacobbe, D.R.; Saffioti, C.; Losito, A.R.; Rinaldi, M.; Aurilio, C.; Bolla, C.; Boni, S.; Borgia, G.; Carannante, N.; Cassola, G.; et al. Use of colistin in adult patients: A cross-sectional study. J. Glob. Antimicrob. Resist. 2020, 20, 43-49. [CrossRef] [PubMed]

12. European Medicines Agency Completes Review of Polymyxin-Based Medicines. Recommendations Issued for Safe Use in Patients with Serious Infections Resistant to Standard Antibiotics. Annex III. Available online: https: / /www.ema.europa.eu/ documents / referral/polymyxin-article-31-referral-annex-iii_en.pdf (accessed on 21 November 2021).

13. Giacobbe, D.R.; Karaiskos, I.; Bassetti, M. How do we optimize the prescribing of intravenous polymyxins to increase their longevity and efficacy in critically ill patients? Expert Opin. Pharm. 2021, 10, 1961743. [CrossRef] [PubMed] 